New Mexico Tech (July 2007)

to be published in the special volume of SIGMA, Proceedings of the 2007 Midwest Geometry Conference in Honor of Thomas P. Branson, Iowa City, IA, May $18-20,2007$

\title{
Heat Kernel Asymptotics on Homogeneous Bundles
}

\author{
Ivan G. Avramidi \\ Department of Mathematics \\ New Mexico Institute of Mining and Technology \\ Socorro, NM 87801, USA \\ Dedicated to the Memory of Thomas P. Branson
}

\begin{abstract}
We consider Laplacians acting on sections of homogeneous vector bundles over symmetric spaces. By using an integral representation of the heat semi-group we find a formal solution for the heat kernel diagonal that gives a generating function for the whole sequence of heat invariants. We argue that the obtained formal solution correctly reproduces the exact heat kernel diagonal after a suitable regularization and analytical continuation.
\end{abstract}

Keywords: Heat Kernel; Symmetric Spaces; Homogeneous Bundles 2000 Mathematics Subject Classification: 58J35; 53C35 


\section{Introduction}

The heat kernel is one of the most powerful tools in mathematical physics and geometric analysis. Of special importance is the short-time asymptotic expansion of the trace of the heat kernel. The coefficients of this asymptotic expansion, called the heat invariants, are extensively used in geometric analysis, in particular, in spectral geometry and index theorems proofs. There has been a tremendous progress in the explicit calculation of spectral asymptotics in the last thirty years $[17,1,12,3,23,26]$ (see also the reviews [18, 8, 10, 24, 20]). A further progress in the study of spectral asymptotics can be only achieved by restricting oneself to operators and manifolds with high level of symmetry, in particular, homogeneous spaces, which enables one to employ powerful algebraic methods. It is well known that heat invariants are determined essentially by local geometry. They are polynomial invariants in the curvature with universal constants that do not depend on the global properties of the manifold. It is this universal structure that we are interested in this paper. Our goal is to compute the heat kernel asymptotics of the Laplacian acting on homogeneous vector bundles over symmetric spaces.

\section{Geometry of Symmetric Spaces}

\subsection{Twisted Spin-Tensor Bundles}

In this section we introduce basic concepts and fix notation. Let $(M, g)$ be an $n$-dimensional Riemannian manifold without boundary. We assume that it is complete simply connected orientable and spin. We denote the local coordinates on $M$ by $x^{\mu}$, with Greek indices running over $1, \ldots, n$. Let $e_{a}{ }^{\mu}$ be a local orthonormal frame defining a basis for the tangent space $T_{x} M$. We denote the frame indices by low case Latin indices from the beginning of the alphabet, which also run over $1, \ldots, n$. The frame indices are raised and lowered by the metric $\delta_{a b}$. Let $e^{a}{ }_{\mu}$ be the matrix inverse to $e_{a}{ }^{\mu}$, defining the dual basis in the cotangent space $T_{x}^{*} M$. As usual, the orthonormal frame, $e^{a}{ }_{\mu}$ and $e_{a}{ }^{\mu}$, will be used to transform the coordinate (Greek) indices to the orthonormal (Latin) indices. The covariant derivative along the frame vectors is defined by $\nabla_{a}=e_{a}{ }^{\mu} \nabla_{\mu}$. For example, with our notation, $\nabla_{a} \nabla_{b} T_{c d}=e_{a}^{\mu} e_{b}{ }^{v} e_{c}^{\alpha} e_{d}^{\beta} \nabla_{\mu} \nabla_{v} T_{\alpha \beta}$.

Let $\mathcal{T}$ be a spin-tensor bundle realizing a representation $\Sigma$ of the spin group $\operatorname{Spin}(n)$ with the fiber $\Lambda$ and let $\Sigma_{a b}$ be the generators of the its Lie algebra. The spin connection naturally induces a connection on the bundle $\mathcal{T}$. The curvature of 
this connection is $\frac{1}{2} R^{a b} \Sigma_{a b}$, where $R^{a b}$ is the curvature 2-form of the spin connection.

In the present paper we will further assume that $M$ is a locally symmetric space with a Riemannian metric with the parallel curvature, that is, $\nabla_{\mu} R_{\alpha \beta \gamma \delta}=0$, which means, in particular, that the Riemann curvature tensor satisfies the integrability constraints

$$
R^{f g}{ }_{e a} R_{b c d}^{e}-R^{f g}{ }_{e b} R_{a c d}^{e}+R^{f g}{ }_{e c}^{e}{ }_{d a b}-R^{f g}{ }_{e d} R_{c a b}^{e}=0 .
$$

Let $G_{Y M}$ be a compact Lie group (called a gauge group). It naturally defines the principal fiber bundle over the manifold $M$ with the structure group $G_{Y M}$. We consider a representation of the structure group $G_{Y M}$ and the associated vector bundle through this representation with the same structure group $G_{Y M}$ whose typical fiber is a $k$-dimensional vector space $W$. Then for any spin-tensor bundle $\mathcal{T}$ we define the twisted spin-tensor bundle $\mathcal{V}$ via the twisted product of the bundles $\mathcal{W}$ and $\mathcal{T}$. The fiber of the bundle $\mathcal{V}$ is $V=\Lambda \otimes W$ so that the sections of the bundle $\mathcal{V}$ are represented locally by $k$-tuples of spin-tensors.

A connection on the bundle $\mathcal{W}$ (called Yang-Mills or gauge connection) taking values in the Lie algebra $\mathcal{G}_{Y M}$ of the gauge group $G_{Y M}$ naturally defines the total connection on the bundle $\mathcal{V}$ with the curvature $\Omega=\frac{1}{2} R^{a b} \Sigma_{a b} \otimes \mathbb{I}_{W}+\mathbb{I}_{\Lambda} \otimes \mathcal{F}$, where $\mathcal{F}$ is the curvature of the Yang-Mills connection.

In the following we will consider homogeneous vector bundles with parallel bundle curvature, that is, $\nabla_{\mu} \mathcal{F}_{\alpha \beta}=0$, which means that the curvature satisfies the integrability constraints

$$
\left[\mathcal{F}_{c d}, \mathcal{F}_{a b}\right]-R^{f}{ }_{a c d} \mathcal{F}_{f b}-R_{b c d}^{f} \mathcal{F}_{a f}=0
$$

\subsection{Normal Coordinates}

Let $x^{\prime}$ be a fixed point in $M$ and $\mathcal{U}$ be a sufficiently small coordinate patch containing the point $x^{\prime}$. Then every point $x$ in $\mathcal{U}$ can be connected with the point $x^{\prime}$ by a unique geodesic. We extend the local orthonormal frame $e_{a}{ }^{\mu}\left(x^{\prime}\right)$ at the point $x^{\prime}$ to a local orthonormal frame $e_{a}^{\mu}(x)$ at the point $x$ by parallel transport. Of course, the frame $e_{a}{ }^{\mu}$ depends on the fixed point $x^{\prime}$ as a parameter. Here and everywhere below the coordinate indices of the tangent space at the point $x^{\prime}$ are denoted by primed Greek letters. They are raised and lowered by the metric tensor $g_{\mu^{\prime} v^{\prime}}\left(x^{\prime}\right)$ at the point $x^{\prime}$. The derivatives with respect to $x^{\prime}$ will be denoted by primed Greek indices as well. 
The parameters of the geodesic connecting the points $x$ and $x^{\prime}$, namely the unit tangent vector at the point $x^{\prime}$ and the length of the geodesic, (or, equivalently, the tangent vector at the point $x^{\prime}$ with the norm equal to the length of the geodesic), provide normal coordinate system for $\mathcal{U}$. Let $d\left(x, x^{\prime}\right)$ be the geodesic distance between the points $x$ and $x^{\prime}$ and $\sigma\left(x, x^{\prime}\right)$ be a two-point function defined by $\sigma\left(x, x^{\prime}\right)=\frac{1}{2}\left[d\left(x, x^{\prime}\right)\right]^{2}$. Then the derivatives $\sigma_{; \mu}\left(x, x^{\prime}\right)$ and $\sigma_{; v^{\prime}}\left(x, x^{\prime}\right)$ are the tangent vectors to the geodesic connecting the points $x$ and $x^{\prime}$ at the points $x$ and $x^{\prime}$ respectively pointing in opposite directions; one is obtained from another by parallel transport. Now, let us define the quantities $y^{a}=e^{a}{ }_{\mu} \sigma^{; \mu}=-e^{a}{ }_{\mu^{\prime}} \sigma^{; \mu^{\prime}}$. These geometric parameters are nothing but the normal coordinates.

Remarks. Two remarks are in order here. First, strictly speaking, normal coordinates can be only defined locally, in geodesic balls of radius less than the injectivity radius of the manifold. However, for symmetric spaces normal coordinates cover the whole manifold except for a set of measure zero where they become singular [14]. This set is precisely the set of points conjugate to the fixed point $x^{\prime}$ and of points that can be connected to the point $x^{\prime}$ by multiple geodesics. In any case, this set is a set of measure zero and, as we will show below, it can be dealt with by some regularization technique. Thus, we will use the normal coordinates defined above for the whole manifold. Second, for compact manifolds (or for manifolds with compact submanifolds) the range of some normal coordinates is also compact, so that if one allows them to range over the whole real line $\mathbb{R}$, then the corresponding compact submanifolds will be covered infinitely many times.

\subsection{Curvature Group of a Symmetric Space}

We assumed that the manifold $M$ is locally symmetric. Since we also assume that it is simply connected and complete, it is a globally symmetric space (or simply symmetric space) [25]. A symmetric space is said to be compact, noncompact or Euclidean if all sectional curvatures are positive, negative or zero. A generic symmetric space has the structure $M=M_{0} \times M_{s}$, where $M_{0}=\mathbb{R}^{n_{0}}$ and $M_{s}=M_{+} \times M_{-}$is a semi-simple symmetric space; it is a product of a compact symmetric space $M_{+}$and a non-sompact symmetric space $M_{-}$. Of course, the dimensions must satisfy the relation $n_{0}+n_{s}=n$, where $n_{s}=\operatorname{dim} M_{s}$.

The components of the curvature tensor can be presented in the form [7]

$$
R_{a b c d}=\beta_{i k} E_{a b}^{i} E_{c d}^{k},
$$

where $\left.E_{a b}^{i}, i=1,2, \ldots, p\right)$, is a collection of $p$ anti-symmetric $n \times n$ matrices and $\beta_{i k}$ is a symmetric nondegenerate $p \times p$ matrix with some $p \leq n(n-1) / 2$. 
In the following the Latin indices from the middle of the alphabet will be used to denote such matrices; they run over $1, \ldots, p$ and should not be confused with the Latin indices from the beginning of the alphabet which denote tensors in $M$. They will be raised and lowered with the matrix $\beta_{i k}$ and its inverse $\left(\beta^{i k}\right)$.

Next, we define the traceless $n \times n$ matrices $D_{i}=\left(D^{a}{ }_{i b}\right)$, where

$$
D^{a}{ }_{i b}=-\beta_{i k} E^{k}{ }_{c b} \delta^{c a} .
$$

The matrices $D_{i}$ are known to be the generators of the holonomy algebra, $\mathcal{H}$, i.e. the Lie algebra of the restricted holonomy group, $H$,

$$
\left[D_{i}, D_{k}\right]=F^{j}{ }_{i k} D_{j},
$$

where $F_{i k}^{j}$ are the structure constants of the holonomy group. The structure constants of the holonomy group define the $p \times p$ matrices $F_{i}$, by $\left(F_{i}\right)^{j}{ }_{k}=F^{j}{ }_{i k}$, which generate the adjoint representation of the holonomy algebra,

$$
\left[F_{i}, F_{k}\right]=F^{j}{ }_{i k} F_{j} .
$$

For symmetric spaces the introduced quantities satisfy additional algebraic constraints. The most important consequence of the eq. (2.1) is the equation [7]

$$
E_{a c}^{i} D_{k b}^{c}-E_{b c}^{i} D_{k a}^{c}=F_{k j}^{i} E^{j}{ }_{a b} .
$$

Now, by using the eqs. (2.5) and (2.7) one can prove that the matrix $\beta_{i k}$ satisfies the equation

$$
\beta_{i k} F^{k}{ }_{j l}+\beta_{l k} F^{k}{ }_{j i}=0 .
$$

Let $h_{b}^{a}$ be the projection to the subspace $T_{x} M_{s}$ of the tangent space of dimension $n_{s}$, that is, the tensor $h_{a b}$ is nothing but the metric tensor on the semi-simple subspace $T_{x} M_{s}$. Since the curvature exists only in the semi-simple submanifold $M_{s}$, the components of the curvature tensor $R_{a b c d}$, as well as the tensors $E_{a b}^{i}$, are non-zero only in the semi-simple subspace $T_{x} M_{s}$. Let $q^{a}{ }_{b}=\delta^{a}{ }_{b}-h^{a}{ }_{b}$ be the projection tensor to the flat subspace $\mathbb{R}^{n_{0}}$. Then

$$
R_{a b c d} q_{e}^{a}=R_{a b} q_{e}^{a}=E_{a b}^{i} q_{e}^{a}=D_{i b}^{a} q_{e}^{b}=D^{a}{ }_{i b} q_{a}^{e}=0 .
$$

Now, we introduce a new type of indices, the capital Latin indices, $A, B, C, \ldots$, which split according to $A=(a, i)$ and run from 1 to $N=p+n$. We define new quantities $C^{A}{ }_{B C}$ by

$$
C^{i}{ }_{a b}=E^{i}{ }_{a b}, \quad C^{a}{ }_{i b}=-C^{a}{ }_{b i}=D^{a}{ }_{i b}, \quad C^{i}{ }_{k l}=F^{i}{ }_{k l},
$$


all other components being zero. Then we can define $N \times N$ matrices $C_{A}$ by $\left(C_{A}\right)^{B}{ }_{C}=C^{B}{ }_{A C}$.

Now, by using the eqs. (2.5), (2.6) and (2.7) one can prove the following theorem [7].

Theorem 1 The matrices $C_{A}$ satisfy the commutation relations

$$
\left[C_{A}, C_{B}\right]=C^{C}{ }_{A B} C_{C},
$$

This means that the matricec $C_{A}$ generate the adjoint representation of a Lie algebra $\mathcal{G}$ with the structure constants $C^{A}{ }_{B C}$. For the lack of a better name we call the algebra $\mathcal{G}$ the curvature algebra. As it will be clear from the next section it is a subalgebra of the total isometry algebra of the symmetric space. It should be clear that the holonomy algebra $\mathcal{H}$ is the subalgebra of the curvature algebra $\mathcal{G}$.

Next, we define a symmetric nondegenerate $N \times N$ matrix

$$
\left(\gamma_{A B}\right)=\left(\begin{array}{cc}
\delta_{a b} & 0 \\
0 & \beta_{i k}
\end{array}\right) \quad \text { and its inverse } \quad\left(\gamma^{A B}\right)=\left(\begin{array}{cc}
\delta^{a b} & 0 \\
0 & \beta^{i k}
\end{array}\right)
$$

These matrices will be used to lower and to raise the capital Latin indices.

Finally, by using the eqs. (2.7) and (2.8) one can show that the matrix $\gamma_{A B}$ satisfies the equation

$$
\gamma_{A B} C^{B}{ }_{C D}+\gamma_{D B} C^{B}{ }_{C A}=0 .
$$

Thus the curvature algebra $\mathcal{G}$ is compact; it is a direct sum of two ideals, $\mathcal{G}=$ $\mathcal{G}_{0} \oplus \mathcal{G}_{s}$, an Abelian center $\mathcal{G}_{0}$ of dimension $n_{0}$ and a semi-simple algebra $\mathcal{G}_{s}$ of dimension $p+n_{s}$.

\subsection{Killing Vectors Fields}

We will use extensively the isometries of the symmetric space $M$. The generators of isometries are the Killing vector fields. The set of all Killing vector fields forms a representation of the isometry algebra, the Lie algebra of the isometry group of the manifold $M$. Let $K=\left(K_{b}^{a}\right)$ be the $n \times n$ matrix with the entries

$$
K_{b}^{a}=R_{c b d}^{a} y^{c} y^{d} .
$$

One can show [7] that

$$
P_{a}=(\sqrt{K} \cot \sqrt{K})^{b}{ }_{a} \frac{\partial}{\partial y^{b}}, \quad L_{i}=-D^{b}{ }_{i a} y^{a} \frac{\partial}{\partial y^{b}},
$$


are Killing vector fields. In the following we will only need these Killing vectors. We introduce the following notation $\left(\xi_{A}\right)=\left(P_{a}, L_{i}\right)$.

Next, by using the explicit form of the Killing vector fields one can prove the following theorem [7].

Theorem 2 The Killing vector fields $\xi_{A}$ satisfy the commutation relations

$$
\left[\xi_{A}, \xi_{B}\right]=C_{A B}^{C} \xi_{C}
$$

Notice that they do not generate the complete isometry algebra of the symmetric space $M$ but rather they form a representation of the curvature algebra $\mathcal{G}$ introduced in the previous section, which is a subalgebra of the total isometry algebra. It is clear that the Killing vector fields $L_{i}$ form a representation of the holonomy algebra $\mathcal{H}$, which is the isotropy algebra of the semi-simple submanifold $M_{s}$, and a subalgebra of the total isotropy algebra of the symmetric space $M$.

We list some properties of the Killing vector fields that will be used below (for details, see [12])

$$
\begin{aligned}
& \gamma^{A B} \xi_{A}{ }^{\mu} \xi_{B}{ }^{v}=g^{\mu v}, \\
& \gamma^{A B} \xi_{A}{ }^{\alpha} \xi_{B}{ }^{\mu} ; \nu \lambda=R_{\lambda \nu \mu}^{\alpha}, \\
& \gamma^{A B} \xi_{A}{ }^{\mu} \xi_{B}{ }^{v} ; \beta=0,
\end{aligned}
$$

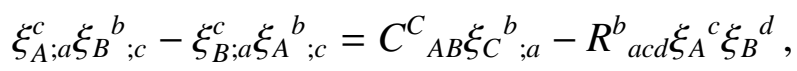

$$
\begin{aligned}
& \gamma^{A B} \xi_{A ; \alpha}{ }^{\mu} \xi_{B}{ }^{v} ; \beta=R_{\alpha \beta}^{\mu}{ }_{\alpha \beta} \text {. }
\end{aligned}
$$

\subsection{Homogeneous Vector Bundles}

Equation (2.2) imposes strong constraints on the curvature of the homogeneous bundle $\mathcal{W}$. We define

$$
\mathcal{B}_{a b}=\mathcal{F}_{c d} q^{c}{ }_{a} q_{b}^{d}, \quad \mathcal{E}_{a b}=\mathcal{F}_{c d} h_{b}^{c} h_{b}^{d},
$$

so that $\mathcal{B}_{a b} h^{a}{ }_{c}=0$, and $\mathcal{E}_{a b} q^{a}{ }_{c}=0$. Then, from eq. (2.2) we obtain

$$
\left[\mathcal{B}_{a b}, \mathcal{B}_{c d}\right]=\left[\mathcal{B}_{a b}, \mathcal{E}_{c d}\right]=0,
$$

and

$$
\left[\mathcal{E}_{c d}, \mathcal{E}_{a b}\right]-R_{a c d}^{f} \mathcal{E}_{f b}-R_{b c d}^{f} \mathcal{E}_{a f}=0
$$


This means that $\mathcal{B}_{a b}$ takes values in an Abelian ideal of the gauge algebra $\mathcal{G}_{Y M}$ and $\mathcal{E}_{a b}$ takes values in the holonomy algebra. More precisely, eq. (2.24) is only possible if the holonomy algebra $\mathcal{H}$ is an ideal of the gauge algebra $\mathcal{G}_{Y M}$. Thus, the gauge group $G_{Y M}$ must have a subgroup $Z \times H$, where $Z$ is an Abelian group and $H$ is the holonomy group.

The matrices $D^{a}{ }_{i b}$ provide a natural embedding of the holonomy algebra $\mathcal{H}$ in the orthogonal algebra $\mathcal{S O}(n)$ in the following sense. Let $X_{a b}$ be the generators of the orthogonal algebra $\mathcal{S O}(n)$ is some representation. Let $T_{i}$ be the matrices defined by

$$
T_{i}=-\frac{1}{2} D^{a}{ }_{i b} X^{b}{ }_{a}
$$

Then one can show that they satisfy the commutation relations

$$
\left[T_{i}, T_{k}\right]=F^{j}{ }_{i k} T_{j}
$$

Thus $T_{i}$ are the generators of the gauge algebra $\mathcal{G}_{Y M}$ realizing a representation $T$ of the holonomy algebra $\mathcal{H}$. Since $\mathcal{B}_{a b}$ takes values in the Abelian ideal of the algebra of the gauge group we also have

$$
\left[\mathcal{B}_{a b}, T_{j}\right]=0 .
$$

Then by using eq. (2.7) one can show that 1

$$
\mathcal{E}_{a b}=\frac{1}{2} R_{a b}^{c d} X_{c d}=-E_{a b}^{i} T_{i}
$$

and

$$
\mathcal{F}_{a b}=-E_{a b}^{i} T_{i}+\mathcal{B}_{a b}=\frac{1}{2} R^{c d}{ }_{a b} X_{c d}+\mathcal{B}_{a b}
$$

Now, we consider the representation $\Sigma$ of the orthogonal algebra defining the spin-tensor bundle $\mathcal{T}$ and define the matrices

$$
G_{a b}=\Sigma_{a b} \otimes \mathbb{I}_{X}+\mathbb{I}_{\Sigma} \otimes X_{a b}
$$

Obviously, these matrices are the generators of the orthogonal algebra in the product representation $\Sigma \otimes X$. Next, the matrices

$$
Q_{i}=-\frac{1}{2} D^{a}{ }_{i b} \Sigma^{b}{ }_{a}
$$

\footnotetext{
${ }^{1}$ We correct here a sign misprint in eq. (3.24) in [7].
} 
form a representation $Q$ of the holonomy algebra $\mathcal{H}$, and the matrices

$$
\mathcal{R}_{i}=Q_{i} \otimes \mathbb{I}_{T}+\mathbb{I}_{\Sigma} \otimes T_{i}=-\frac{1}{2} D^{a}{ }_{i b} G^{b}{ }_{a}
$$

are the generators of the holonomy algebra in the product representation $\mathcal{R}=$ $Q \otimes T$. Then the total curvature of a twisted spin-tensor bundle $\mathcal{V}$ is

$$
\Omega_{a b}=-E_{a b}^{i} \mathcal{R}_{i}+\mathcal{B}_{a b}=\frac{1}{2} R^{c d}{ }_{a b} G_{c d}+\mathcal{B}_{a b}
$$

\subsection{Twisted Lie Derivatives}

Let $\varphi$ be a section of a twisted homogeneous spin-tensor bundle $\mathcal{T}$. Let $\xi_{A}$ be the basis of Killing vector fields. Then the covariant (or generalized, or twisted) Lie derivative of $\varphi$ along $\xi_{A}$ is defined by

$$
\mathcal{L}_{A} \varphi=\mathcal{L}_{\xi_{A}} \varphi=\left(\nabla_{\xi_{A}}+S_{A}\right) \varphi
$$

where $\nabla_{\xi_{A}}=\xi_{A}{ }^{\mu} \nabla_{\mu}$, and $S_{A}=\frac{1}{2} \xi_{A ; b}{ }^{a} G^{b}{ }_{a}$. Note that $S_{a} q_{b}^{a}=0$.

\section{Proposition 1 There hold}

$$
\begin{gathered}
{\left[\nabla_{\xi_{A}}, \nabla_{\xi_{B}}\right] \varphi=\left(C^{C}{ }_{A B} \nabla_{\xi_{C}}-\mathcal{R}_{A B}+\mathcal{B}_{A B}\right) \varphi} \\
\nabla_{\xi_{A}} S_{B}=\mathcal{R}_{A B} \\
{\left[S_{A}, S_{B}\right]=C^{C}{ }_{A B} S_{C}-\mathcal{R}_{A B}}
\end{gathered}
$$

where

$$
\begin{gathered}
\mathcal{R}_{A B}=\xi_{A}{ }^{a} \xi_{B}{ }^{b} E^{i}{ }_{a b} \mathcal{R}_{i}=-\frac{1}{2} R^{c d}{ }_{a b} \xi_{A}{ }^{a} \xi_{B}{ }^{b} G_{c d}, \\
\mathcal{B}_{A B}=\xi_{A}{ }^{a} \xi_{B}{ }^{b} \mathcal{B}_{a b} .
\end{gathered}
$$

Proof. By using the properties of the Killing vectors described in the previous section and the eq. (2.33) we obtain first (2.35). Next, we obtain (2.36), and, further, by using the eq. (2.20) we get (2.37).

We define the operator

$$
\mathcal{L}^{2}=\gamma^{A B} \mathcal{L}_{A} \mathcal{L}_{B} .
$$


Theorem 3 The operators $\mathcal{L}_{A}$ and $\mathcal{L}^{2}$ satisfy the commutation relations

$$
\begin{gathered}
{\left[\mathcal{L}_{A}, \mathcal{L}_{B}\right]=C^{C}{ }_{A B} \mathcal{L}_{C}+\mathcal{B}_{A B},} \\
{\left[\mathcal{L}_{A}, \mathcal{L}^{2}\right]=2 \gamma^{B C} \mathcal{B}_{A B} \mathcal{L}_{C} .}
\end{gathered}
$$

Proof. This follows from

$$
\left[\mathcal{L}_{A}, \mathcal{L}_{B}\right]=\left[\nabla_{\xi_{A}}, \nabla_{\xi_{B}}\right]+\left[\nabla_{\xi_{A}}, S_{B}\right]-\left[\nabla_{\xi_{B}}, S_{A}\right]+\left[S_{A}, S_{B}\right]
$$

and eqs. (2.35), (2.36), and (2.37). The eq. (2.42) follows directly from (2.41).

The operators $\mathcal{L}_{A}$ form an algebra that is a direct sum of a nilpotent ideal and a semisimple algebra. For the lack of a better name we call this algebra gauged curvature algebra and denote it by $\mathcal{G}_{\text {gauge }}$.

Now, by using the eqs. (2.19), (2.36) and (2.18) one can prove that

$$
\gamma^{A B} \xi_{A}{ }^{\mu} S_{B}=0, \quad \gamma^{A B} \nabla_{\xi_{A}} S_{B}=0, \quad \gamma^{A B} S_{A} S_{B}=\mathcal{R}^{2}
$$

We define the Casimir operator

$$
\mathcal{R}^{2}=\beta^{i j} \mathcal{R}_{i} \mathcal{R}_{j}=\frac{1}{4} R^{a b c d} G_{a b} G_{c d} .
$$

Theorem 4 The Laplacian $\Delta$ acting on sections of a twisted spin-tensor bundle $\mathcal{V}$ over a symmetric space has the form

$$
\Delta=\mathcal{L}^{2}-\mathcal{R}^{2}
$$

Therefore,

$$
\left[\mathcal{L}_{A}, \Delta\right]=2 \gamma^{B C} \mathcal{B}_{A B} \mathcal{L}_{C}
$$

Proof. We have

$$
\gamma^{A B} \mathcal{L}_{A} \mathcal{L}_{B}=\gamma^{A B} \nabla_{\xi_{A}} \nabla_{\xi_{B}}+\gamma^{A B} S_{A} \nabla_{\xi_{B}}+\gamma^{A B} \nabla_{\xi_{A}} S_{B}+\gamma^{A B} S_{A} S_{B}
$$

Now, by using eqs. (2.17) and (2.19) we get

$$
\gamma^{A B} \nabla_{\xi_{A}} \nabla_{\xi_{B}}=\Delta
$$

Next, by using the eqs. (2.36) and (2.44) we obtain (2.46). The eq. (2.47) follows from the commutation relations (2.41). 


\subsection{Isometries and Pullbacks}

Let $\omega^{i}$ be the canonical coordinates on the holonomy group and $\left(k^{A}\right)=\left(p^{a}, \omega^{i}\right)$ be the canonical coordinates on the gauged curvature group. Let $\xi=\langle k, \xi\rangle=k^{A} \xi_{A}=$ $p^{a} P_{a}+\omega^{i} L_{i}$ be a Killing vector field and let $\psi_{t}: M \rightarrow M$ be the one-parameter diffeomorphism (the isometry) generated by the vector field $\xi$. Let $\hat{x}=\psi_{t}(x)$, so that $\frac{d \hat{x}}{d t}=\xi(\hat{x})$ and $\left.\hat{x}\right|_{t=0}=x$. The solution of this equation $\hat{x}=\hat{x}\left(t, p, \omega, x, x^{\prime}\right)$. depends on the parameters $t, p, \omega, x$ and $x^{\prime}$. We will be interested mainly in the case when the points $x$ and $x^{\prime}$ are close to each other. In fact, at the end of our calculations we will take the limit $x=x^{\prime}$.

Now, we choose the normal coordinates $y^{a}$ of the point defined above and the normal coordinates $\hat{y}^{a}$ of the point $\hat{x}$ with the origin at $x^{\prime}$, so that the normal coordinates $y^{\prime}$ of the point $x^{\prime}$ are equal to zero. Recall that the normal coordinates are equal to the components of the tangent vector at the point $x^{\prime}$ to the geodesic connecting the points $x^{\prime}$ and the current point, that is, $y^{a}=-e_{\mu^{\prime}}^{a}\left(x^{\prime}\right) \sigma^{; \mu^{\prime}}\left(x, x^{\prime}\right)$ and $\hat{y}^{a}=-e^{a}{ }_{\mu^{\prime}}\left(x^{\prime}\right) \sigma^{; \mu^{\prime}}\left(\hat{x}, x^{\prime}\right)$. Then by taking into account the explicit form of the Killing vectors given by eq. (2.15) we have

$$
\frac{d \hat{y}^{a}}{d t}=(\sqrt{K(\hat{y})} \cot \sqrt{K(\hat{y})})_{b}^{a} p^{b}-\omega^{i} D^{a}{ }_{i b} \hat{y}^{b},
$$

with the initial condition $\left.\hat{y}^{a}\right|_{t=0}=y^{a}$. The solution of this equation defines a function $\hat{y}=\hat{y}(t, p, \omega, y)$.

We define the matrix

$$
D(\omega)=\omega^{i} D_{i}
$$

Proposition 2 The Taylor expansion of the function $\hat{y}=\hat{y}(t, p, \omega, y)$ in $p$ and $y$ reads

$$
\hat{y}^{a}=(\exp [-t D(\omega)])^{a}{ }_{b} y^{b}+\left(\frac{1-\exp [-t D(\omega)]}{D(\omega)}\right)^{a}{ }_{b} p^{b}+O\left(y^{2}, p^{2}, p y\right) .
$$

There holds

$$
\left.\operatorname{det}\left(\frac{\partial \hat{y}^{a}}{\partial p^{b}}\right)\right|_{p=y=0, t=1}=\operatorname{det}_{T M}\left(\frac{\sinh [D(\omega) / 2]}{D(\omega) / 2}\right) .
$$

Proof. First, for $p=0$ from eq. (2.50) we obtain $\hat{y}(t, 0, \omega, 0)=0$. Next, by differentiating the eq. (2.50) with respect to $y^{b}$ and setting $p=y=0$ we obtain

$$
\left.\frac{\partial \hat{y}^{a}}{\partial y^{b}}\right|_{p=y=0}=(\exp [-t D(\omega)])^{a}
$$


Further, by differentiating the eq. (2.50) with respect to $p^{b}$ and setting $p=0$, we obtain a differential equation for the matrix $\left.\frac{\partial \hat{y}^{a}}{\partial p^{b}}\right|_{p=y=0}$ whose solution is

$$
\left.\left(\frac{\partial \hat{y}^{a}}{\partial p^{b}}\right)\right|_{p=y=0}=\frac{1-\exp [-t D(\omega)]}{D(\omega)} .
$$

By using the obtained results we get the desired formula (2.52). Finally, by taking into account that the matrix $D(\omega)$ is traceless, by using eq. (2.55) we obtain (2.53).

The function $\hat{y}=\hat{y}(t, p, \omega, y)$ implicitly defines the function $p=p(t, \omega, \hat{y}, y)$. The function $\bar{p}=\bar{p}(\omega, y)$ is now defined by the equation $\hat{y}(1, \bar{p}, \omega, y)=0$, or $\bar{p}(\omega, y)=p(1, \omega, 0, y)$.

Proposition 3 The Taylor expansion of the function $\bar{p}(\omega, y)$ in y has the form

$$
\bar{p}^{a}=-\left(D(\omega) \frac{\exp [-D(\omega)]}{1-\exp [-D(\omega)]}\right)^{a}{ }_{b} y^{b}+O\left(y^{2}\right) .
$$

Therefore,

$$
\left.\operatorname{det}\left(-\frac{\partial \bar{p}^{a}}{\partial y^{b}}\right)\right|_{y=0}=\operatorname{det}_{T M}\left(\frac{\sinh [D(\omega) / 2]}{D(\omega) / 2}\right)^{-1} .
$$

Proof. Next, by taking into account the eq. $\left.\hat{y}\right|_{p=y=0}=0$ we have $\left.\bar{p}\right|_{y=0}=0$. Further, by differentiating the equation $\hat{y}(1, \bar{p}, \omega, y)=0$ with respect to $y^{c}$ and setting $y=0$ we get

$$
\left.\frac{\partial \hat{y}^{a}}{\partial y^{b}}\right|_{p=y=0, t=1}+\left.\left.\frac{\partial \hat{y}^{a}}{\partial p^{c}}\right|_{p=y=0, t=1} \frac{\partial \bar{p}^{c}}{\partial y^{b}}\right|_{y=0}=0,
$$

and, therefore,

$$
\left.\frac{\partial \bar{p}^{a}}{\partial y^{b}}\right|_{y=0}=-\left(D(\omega) \frac{\exp [-D(\omega)]}{1-\exp [-D(\omega)]}\right)^{a} .
$$

This leads to both (2.56) and (2.57).

Now, we define

$$
\Lambda_{\nu}^{\hat{\mu}}=\frac{\partial \hat{x}^{\mu}}{\partial x^{\nu}}, \quad \text { so that } \quad\left(\Lambda^{-1}\right)^{\mu}{ }_{\hat{\alpha}}=g^{\mu \nu}(x) \Lambda^{\hat{\beta}} g_{\hat{\beta} \hat{\alpha}}(\hat{x}) .
$$


Let $e^{a}{ }_{\mu}$ and $e_{a}{ }^{\mu}$ be a local orthonormal frame that is obtained by parallel transport along geodesics from a point $x^{\prime}$ and $\psi_{t}^{*}$ be the pullback of the isometry $\psi_{t}$ defined above. Then the frames of 1-forms $e^{a}$ and $\psi_{t}^{*} e^{a}$ are related by an orthogonal transformation $\left(\psi_{t}^{*} e^{a}\right)(x)=O^{a}{ }_{b} e^{b}(x)$, where the matrix $O^{a}{ }_{b}$ is defined by

$$
O^{a}{ }_{b}=e_{\hat{\alpha}}^{a}(\hat{x}) \Lambda^{\hat{\alpha}}{ }_{\mu} e_{b}{ }^{\mu}(x) .
$$

Since the matrix $O$ is orthogonal, it can be parametrized by $O=\exp \theta$, where $\theta_{a b}$ is an antisymmetric matrix.

Proposition 4 For $p=y=0$ the matrix $O$ has the form

$$
\left.O\right|_{p=y=0}=\exp [-t D(\omega)] .
$$

Proof. We use normal coordinates $\hat{y}^{a}$ and $y^{a}$. Then the matrix $O$ takes the form

$$
O_{b}^{a}=e^{a}{ }_{\hat{\alpha}} \frac{\partial \hat{x}^{\alpha}}{\partial \hat{y}^{c}} \frac{\partial \hat{y}^{c}}{\partial y^{d}} \frac{\partial y^{d}}{\partial x^{\mu}} e_{b}{ }^{\mu} .
$$

Now, by using the explicit form of the Jacobian in normal coordinates and the fact that $\left.\hat{y}\right|_{p=y=0}=0$ we obtain

$$
\left.\frac{\partial y^{a}}{\partial x^{\mu}} e_{b}{ }^{\mu}\right|_{p=y=0}=\left.e^{a}{ }_{\hat{\alpha}} \frac{\partial \hat{x}^{\alpha}}{\partial \hat{y}^{b}}\right|_{p=y=0}=\delta^{a}{ }_{b} .
$$

Therefore,

$$
\left.O^{a}\right|_{p=y=0}=\left.\frac{\partial \hat{y}^{a}}{\partial y^{b}}\right|_{p=y=0},
$$

and, finally (2.54) gives the desired result (2.62).

Let $\varphi$ be a section of the twisted spin-tensor bundle $\mathcal{V}$. Let $V_{x}$ be the fiber at the point $x$ and $V_{\hat{x}}$ be the fiber at the point $\hat{x}=\psi_{t}(x)$. The pullback of the diffeomorphism $\psi_{t}$ defines the map, that we call just the pullback, $\psi_{t}^{*}: C^{\infty}(\mathcal{V}) \rightarrow$ $C^{\infty}(\mathcal{V})$ on smooth sections of the twisted spin-tensor bundle $\mathcal{V}$.

Proposition 5 Let $\varphi$ be a section of a twisted spin-tensor bundle $\mathcal{V}$. Then

$$
\left(\psi_{t}^{*} \varphi\right)(x)=\exp \left(-\frac{1}{2} \theta_{a b} G^{a b}\right) \varphi(\hat{x}) .
$$


In particular, for $p=y=0\left(\right.$ or $\left.x=x^{\prime}\right)$

$$
\left.\left(\psi_{t}^{*} \varphi\right)(x)\right|_{p=y=0}=\exp [t \mathcal{R}(\omega)] \varphi\left(x^{\prime}\right),
$$

where $\mathcal{R}(\omega)=\omega^{i} \mathcal{R}_{i}$

Proof. First, from the eq. (2.62) we see that $\left.\theta^{a}\right|_{p=y=0}=-t \omega^{i} D^{a}{ }_{i b}$. Then, from the definition (2.32) of the matrices $\mathcal{R}_{i}$ we get (2.67).

\section{Heat Semigroup}

\subsection{Geometry of the Curvature Group}

Let $G_{\text {gauge }}$ be the gauged curvature group and $H$ be its holonomy subgroup. Both these groups have compact algebras. However, while the holonomy group is always compact, the curvature group is, in general, a product of a nilpotent group, $G_{0}$, and a semi-simple group, $G_{s}, G_{\text {gauge }}=G_{0} \times G_{s}$. The semi-simple group $G_{s}$ is a product $G_{s}=G_{+} \times G_{-}$of a compact $G_{+}$and a non-compact $G_{-}$subgroups.

Let $\xi_{A}$ be the basis Killing vectors, $k^{A}$ be the canonical coordinates on the curvature group $G$ and $\xi(k)=k^{A} \xi_{A}$. The canonical coordinates are exactly the normal coordinates on the group defined above. Let $C_{A}$ be the generators of the curvature group in adjoint representation and $C(k)=k^{A} C_{A}$. In the following $\partial_{M}$ means the partial derivative $\partial / \partial k^{M}$ with respect to the canonical coordinates. We define the matrix $Y^{A}{ }_{M}$ by the equation

$$
\exp [-\xi(k)] \partial_{M} \exp [\xi(k)]=Y^{A}{ }_{M} \xi_{A},
$$

which is well defined since the right hand side lies in the Lie algebra of the curvature group. The matrix $Y=\left(Y^{A}{ }_{M}\right)$ can be computed explicitly, namely,

$$
Y=\frac{1-\exp [-C(k)]}{C(k)} .
$$

Let $X=\left(X_{A}{ }^{M}\right)=Y^{-1}$ be the inverse matrix of $Y$. Then we define the 1-forms $Y^{A}$ and the vector fields $X_{A}$ on the group $G$ by

$$
Y^{A}=Y^{A}{ }_{M} d k^{M}, \quad X_{A}=X_{A}{ }^{M} \partial_{M} .
$$


Proposition 6 There holds

$$
X_{A} \exp [\xi(k)]=\exp [\xi(k)] \xi_{A} .
$$

Proof. This follows immediately from the eq. (3.1).

Next, by differentiating the eq. (3.1) with respect to $k^{L}$ and alternating the indices $L$ and $M$ we obtain

$$
\partial_{L} Y^{A}{ }_{M}-\partial_{M} Y^{A}{ }_{L}=-C_{B C}^{A} Y^{B}{ }_{L} Y^{C}{ }_{M},
$$

which, of course, can also be written as

$$
d Y^{A}=-\frac{1}{2} C^{A}{ }_{B C} Y^{B} \wedge Y^{C}
$$

Proposition 7 The vector fields $X_{A}$ satisfy the commutation relations

$$
\left[X_{A}, X_{B}\right]=C_{A B}^{C} X_{C}
$$

Proof. This follows from the eq. (3.5).

The vector fields $X_{A}$ are nothing but the right-invariant vector fields. They form a representation of the curvature algebra.

We will also need the following fundamental property of Lie groups.

Proposition 8 Let $G$ be a Lie group with the structure constants $C^{A}{ }_{B C}, C_{A}=$ $\left(C^{B}{ }_{A C}\right)$ and $C(k)=C_{A} k^{A}$. Let $\gamma=\left(\gamma_{A B}\right)$ be a symmetric non-degenerate matrix satisfying the equation

$$
\left(C_{A}\right)^{T}=-\gamma C_{A} \gamma^{-1}
$$

Let $X=\left(X_{A}{ }^{M}\right)$ be a matrix defined by

$$
X=\frac{C(k)}{1-\exp [-C(k)]} .
$$

Then

$$
(\operatorname{det} X)^{-1 / 2} \gamma^{A B} X_{A}{ }^{M} \partial_{M} X_{B}{ }^{N} \partial_{N}(\operatorname{det} X)^{1 / 2}=-\frac{1}{24} \gamma^{A B} C^{C}{ }_{A D} C^{D}{ }_{B C}
$$


Proof. It is easy to check that this equation holds at $k=0$. Now, it can be proved by showing that it is a group invariant. For a detailed proof for semisimple groups see [19, 14, 16].

It is worth stressing that this equation holds not only on semisimple Lie groups but on any group with a compact Lie algebra, that is, when the structure constants $C_{B C}^{A}$ and the matrix $\gamma_{A B}$, used to define the metric $G_{M N}$ and the operator $X^{2}$, satisfy the eq. (2.13). Such algebras can have an Abelian center.

Now, by using the right-invariant vector fields we define a metric on the curvature group $G$

$$
G_{M N}=\gamma_{A B} Y^{A}{ }_{M} Y^{B}{ }_{N}, \quad G^{M N}=\gamma^{A B} X_{A}{ }^{M} X_{B}{ }^{N} .
$$

This metric is bi-invariant. This means that the vector fields $X_{A}$ are the Killing vector fields of the metric $G_{M N}$. One can easily show that this metric defines the following natural affine connection $\nabla^{G}$ on the group

$$
\nabla_{X_{C}}^{G} X_{A}=-\frac{1}{2} C^{A}{ }_{B C} X_{B}, \quad \nabla_{X_{C}}^{G} Y^{A}=\frac{1}{2} C^{B}{ }_{A C} Y^{B},
$$

with the scalar curvature

$$
R_{G}=-\frac{1}{4} \gamma^{A B} C^{C}{ }_{A D} C^{D}{ }_{B C} .
$$

Since the matrix $C(k)$ is traceless we have $\operatorname{det} \exp [C(k) / 2]=1$, and, therefore, the volume element on the group is

$$
|G|^{1 / 2}=\left(\operatorname{det} G_{M N}\right)^{1 / 2}=|\gamma|^{1 / 2} \operatorname{det}_{\mathcal{G}}\left(\frac{\sinh [C(k) / 2]}{C(k) / 2}\right),
$$

where $|\gamma|=\operatorname{det} \gamma_{A B}$.

It is not difficult to see that

$$
k^{M} Y^{A}{ }_{M}=k^{M} X_{M}{ }^{A}=k^{A} .
$$

By differentiating this equation with respect to $k^{B}$ and contracting the indices $A$ and $B$ we obtain

$$
k^{M} \partial_{A} X_{M}{ }^{A}=N-X_{A}{ }^{A} .
$$

Now, by contracting the eq. (3.12) with $G^{B C}$ we obtain the zero-divergence condition for the right-invariant vector fields

$$
|G|^{-1 / 2} \partial_{M}\left(|G|^{1 / 2} X_{A}^{M}\right)=0 .
$$


Next, we define the Casimir operator

$$
X^{2}=C_{2}(G, X)=\gamma^{A B} X_{A} X_{B} .
$$

By using the eq. (3.17) one can easily show that $X^{2}$ is an invariant differential operator that is nothing but the scalar Laplacian on the group

$$
X^{2}=|G|^{-1 / 2} \partial_{M}|G|^{1 / 2} G^{M N} \partial_{N}=G^{M N} \nabla_{M}^{G} \nabla_{N}^{G} .
$$

Then, by using the eqs. (2.13) and (2.11) one can show that the operator $X^{2}$ commutes with the operators $X_{A}$,

$$
\left[X_{A}, X^{2}\right]=0 .
$$

Since we will actually be working with the gauged curvature group, we introduce now the operators (covariant right-invariant vector fields) $J_{A}$ by

$$
J_{A}=X_{A}-\frac{1}{2} \mathcal{B}_{A B} k^{B},
$$

and the operator

$$
J^{2}=\gamma^{A B} J_{A} J_{B}
$$

Proposition 9 The operators $J_{A}$ and $J^{2}$ satisfy the commutation relations

$$
\left[J_{A}, J_{B}\right]=C^{C}{ }_{A B} J_{C}+\mathcal{B}_{A B}
$$

and

$$
\left[J_{A}, J^{2}\right]=2 \mathcal{B}_{A B} J^{B}
$$

Proof. By using the eq. (2.22) we obtain

$$
X_{B}{ }^{A} \mathcal{B}_{A M}=\gamma_{B N} \gamma^{A C} X_{C}{ }^{N} \mathcal{B}_{A M}=\mathcal{B}_{B M},
$$

and, hence,

$$
\gamma^{A B} X_{B}{ }^{M} \mathcal{B}_{A M}=0
$$

and, further, by using (3.7) we obtain (3.23). By using the eqs. (3.25) we get (3.24).

Thus, the operators $J_{A}$ form a representation of the gauged curvature algebra. Now, let $\mathcal{L}_{A}$ be the operators of Lie derivatives satisfying the commutation relations (2.41) and $\mathcal{L}(k)=k^{A} \mathcal{L}_{A}$. 
Proposition 10 There holds

$$
J_{A} \exp [\mathcal{L}(k)]=\exp [\mathcal{L}(k)] \mathcal{L}_{A}
$$

and, therefore,

$$
J^{2} \exp [\mathcal{L}(k)]=\exp [\mathcal{L}(k)] \mathcal{L}^{2}
$$

Proof. We have

$$
\exp [-\mathcal{L}(k)] \partial_{M} \exp [\mathcal{L}(k)]=\exp \left[-A d_{\mathcal{L}(k)}\right] \partial_{M}
$$

By using the commutation relations (2.41) and eq. (2.22) we obtain

$$
\exp [-\mathcal{L}(k)] \partial_{M} \exp [\mathcal{L}(k)]=Y^{A}{ }_{M} \mathcal{L}_{A}+\frac{1}{2} \mathcal{B}_{M N} k^{N} .
$$

The statement of the proposition follows from the definition of the operators $J_{A}$, $J^{2}$ and $\mathcal{L}^{2}$.

\subsection{Heat Kernel on the Curvature Group}

Let $\mathcal{B}$ be the matrix with the components $\mathcal{B}=\left(\gamma^{A B} \mathcal{B}_{B C}\right)$ so that

$$
\mathcal{B}=\left(\mathcal{B}_{A B}\right)=\left(\begin{array}{cc}
\mathcal{B}_{a b} & 0 \\
0 & 0
\end{array}\right)
$$

Let $k^{A}$ be the canonical coordinates on the curvature group $G$ and $A(t ; k)$ be a function defined by

$$
A(t ; k)=\operatorname{det}_{\mathcal{G}}\left(\frac{\sinh [C(k) / 2+t \mathcal{B}]}{C(k) / 2+t \mathcal{B}}\right)^{-1 / 2} .
$$

By using the eqs. (3.25) one can rewrite this in the form

$$
A(t ; k)=\operatorname{det}_{\mathcal{G}}\left(\frac{\sinh [C(k) / 2]}{C(k) / 2}\right)^{-1 / 2} \operatorname{det}_{\mathcal{G}}\left(\frac{\sinh [t \mathcal{B}]}{t \mathcal{B}}\right)^{-1 / 2}
$$

Notice also that

$$
\operatorname{det}_{\mathcal{G}}\left(\frac{\sinh [t \mathcal{B}]}{t \mathcal{B}}\right)^{-1 / 2}=\operatorname{det}_{T M}\left(\frac{\sinh [t \mathcal{B}]}{t \mathcal{B}}\right)^{-1 / 2},
$$


where $\mathcal{B}$ is now regarded as just the matrix $\mathcal{B}=\left(\mathcal{B}^{a}{ }_{b}\right)$.

Let $\Theta(t ; k)$ be another function on the group $G$ defined by

$$
\Theta(t ; k)=\frac{1}{2}\langle k, \gamma \hat{\Theta} k\rangle,
$$

where $\hat{\Theta}$ is the matrix

$$
\hat{\Theta}=t \mathcal{B} \operatorname{coth}(t \mathcal{B})
$$

and $\langle u, \gamma v\rangle=\gamma_{A B} u^{A} v^{B}$ is the inner product on the algebra $\mathcal{G}$.

Theorem 5 Let $\Phi(t ; k)$ be a function on the group $G$ defined by

$$
\Phi(t ; k)=(4 \pi t)^{-N / 2} A(t ; k) \exp \left(-\frac{\Theta(t ; k)}{2 t}+\frac{1}{6} R_{G} t\right),
$$

Then $\Phi(t ; k)$ satisfies the equation

$$
\partial_{t} \Phi=J^{2} \Phi
$$

and the initial condition

$$
\Phi(0 ; k)=|\gamma|^{-1 / 2} \delta(k) .
$$

Proof. We compute first

$$
\partial_{t} \Phi=\left[\frac{1}{6} R_{G}-\frac{1}{2 t} \operatorname{tr}_{\mathcal{G}} \hat{\Theta}+\frac{1}{4 t^{2}}\left\langle k, \gamma \hat{\Theta}^{2} k\right\rangle-\frac{1}{4}\left\langle k, \gamma \mathcal{B}^{2} k\right\rangle\right] \Phi .
$$

Next, we have

$$
J^{2}=X^{2}-\gamma^{A B} \mathcal{B}_{A C} k^{C} X_{B}+\frac{1}{4} \gamma^{A B} \mathcal{B}_{A C} \mathcal{B}_{B D} k^{C} k^{D}
$$

By using the eqs. (3.25) and (3.31) and the anti-symmetry of the matrix $\mathcal{B}_{A B}$ we show that

$$
\mathcal{B}_{A C} k^{C} X_{B} \Phi=0
$$

Thus,

$$
\begin{aligned}
J^{2} \Phi= & {\left[A^{-1}\left(X^{2} A\right)-\frac{1}{2 t}\left(X^{2} \Theta\right)+\frac{1}{4 t^{2}} \gamma^{A B}\left(X_{A} \Theta\right)\left(X_{B} \Theta\right)\right.} \\
& \left.-\frac{1}{t} A^{-1} \gamma^{A B}\left(X_{B} A\right)\left(X_{A} \Theta\right)-\frac{1}{4}\left\langle k, \gamma \mathcal{B}^{2} k\right\rangle\right] \Phi
\end{aligned}
$$


Further, by using 3.25) we get

$$
\gamma^{A B}\left(X_{A} \Theta\right)\left(X_{B} \Theta\right)=\left\langle k, \gamma \hat{\Theta}^{2} k\right\rangle, \quad X^{2} \Theta=\operatorname{tr}_{\mathcal{G}} X+\operatorname{tr}_{\mathcal{G}} \hat{\Theta}-N
$$

Now, by using the eq. (3.17) and eqs. (3.31) and (3.16) we show that

$$
A^{-1} \gamma^{A B}\left(X_{A} \Theta\right) X_{B} A=\frac{1}{2}\left(N-\operatorname{tr}_{\mathcal{G}} X\right),
$$

and by using eq. (3.10) we obtain

$$
A^{-1} X^{2} A=\frac{1}{6} R_{G}
$$

Finally, substituting the eqs. (3.44)-(3.46) into eq. (3.43) and comparing it with eq. (3.40) we prove the eq. (3.38). The initial condition (3.39) follows easily from the well known property of the Gaussian. This completes the proof of the theorem.

\subsection{Regularization and Analytical Continuation}

In the following we will complexify the gauged curvature group in the following sense. We extend the canonical coordinates $\left(k^{A}\right)=\left(p^{a}, \omega^{i}\right)$ to the whole complex Euclidean space $\mathbb{C}^{N}$. Then all group-theoretic functions introduced above become analytic functions of $k^{A}$ possibly with some poles on the real section $\mathbb{R}^{N}$ for compact groups. In fact, we replace the actual real slice $\mathbb{R}^{N}$ of $\mathbb{C}^{N}$ with an $N$-dimensional subspace $\mathbb{R}_{\text {reg }}^{N}$ in $\mathbb{C}^{N}$ obtained by rotating the real section $\mathbb{R}^{N}$ counterclockwise in $\mathbb{C}^{N}$ by $\pi / 4$. That is, we replace each coordinate $k^{A}$ by $e^{i \pi / 4} k^{A}$. In the complex domain the group becomes non-compact. We call this procedure the decompactification. If the group is compact, or has a compact subgroup, then this plane will cover the original group infinitely many times.

Since the metric $\left(\gamma_{A B}\right)=\operatorname{diag}\left(\delta_{a b}, \beta_{i j}\right)$ is not necessarily positive definite, (actually, only the metric of the holonomy group $\beta_{i j}$ is non-definite) we analytically continue the function $\Phi(t ; k)$ in the complex plane of $t$ with a cut along the negative imaginary axis so that $-\pi / 2<\arg t<3 \pi / 2$. Thus, the function $\Phi(t ; k)$ defines an analytic function of $t$ and $k^{A}$. For the purpose of the following exposition we shall consider $t$ to be real negative, $t<0$. This is needed in order to make all integrals convergent and well defined and to be able to do the analytical continuation.

As we will show below, the singularities occur only in the holonomy group. This means that there is no need to complexify the coordinates $p^{a}$. Thus, in the 
following we assume the coordinates $p^{a}$ to be real and the coordinates $\omega^{i}$ to be complex, more precisely, to take values in the $p$-dimensional subspace $\mathbb{R}_{\text {reg }}^{p}$ of $\mathbb{C}^{p}$ obtained by rotating $\mathbb{R}^{p}$ counterclockwise by $\pi / 4$ in $\mathbb{C}^{p}$ That is, we have $\mathbb{R}_{\mathrm{reg}}^{N}=$ $\mathbb{R}^{n} \times \mathbb{R}_{\text {reg }}^{p}$.

This procedure (that we call a regularization) with the nonstandard contour of integration is necessary for the convergence of the integrals below since we are treating both the compact and the non-compact symmetric spaces simultaneously. Recall, that, in general, the nondegenerate diagonal matrix $\beta_{i j}$ is not positive definite. The space $\mathbb{R}_{\text {reg }}^{p}$ is chosen in such a way to make the Gaussian exponent purely imaginary. Then the indefiniteness of the matrix $\beta$ does not cause any problems. Moreover, the integrand does not have any singularities on these contours. The convergence of the integral is guaranteed by the exponential growth of the sine for imaginary argument. These integrals can be computed then in the following way. The coordinates $\omega^{j}$ corresponding to the compact directions are rotated further by another $\pi / 4$ to imaginary axis and the coordinates $\omega^{j}$ corresponding to the non-compact directions are rotated back to the real axis. Then, for $t<0$ all the integrals below are well defined and convergent and define an analytic function of $t$ in a complex plane with a cut along the negative imaginary axis.

\subsection{Heat Semigroup}

Theorem 6 The heat semigroup $\exp \left(t \mathcal{L}^{2}\right)$ can be represented in form of the integral

$$
\exp \left(t \mathcal{L}^{2}\right)=\int_{\mathbb{R}_{\mathrm{reg}}^{N}} d k|G|^{1 / 2}(k) \Phi(t ; k) \exp [\mathcal{L}(k)]
$$

Proof. Let

$$
\Psi(t)=\int_{\mathbb{R}_{\mathrm{rgg}}^{N}} d k|G|^{1 / 2} \Phi(t ; k) \exp [\mathcal{L}(k)] .
$$

By using the previous theorem we obtain

$$
\partial_{t} \Psi(t)=\int_{\mathbb{R}_{\mathrm{reg}}^{N}} d k|G|^{1 / 2} \exp [\mathcal{L}(k)] J^{2} \Phi(t ; k) .
$$

Now, by integrating by parts we get

$$
\partial_{t} \Psi(t)=\int_{\mathbb{R}_{\mathrm{reg}}^{N}} d k|G|^{1 / 2} \Phi(t ; k) J^{2} \exp [\mathcal{L}(k)],
$$


and, by using eq. (3.28) we obtain

$$
\partial_{t} \Psi(t)=\Psi(t) \mathcal{L}^{2} .
$$

Finally from the initial condition (3.39) for the function $\Phi(t ; k)$ we get $\Psi(0)=1$, and, therefore, $\Psi(t)=\exp \left(t \mathcal{L}^{2}\right)$.

Theorem 7 Let $\Delta$ be the Laplacian acting on sections of a homogeneous twisted spin-tensor vector bundle over a symmetric space. Then the heat semigroup $\exp (t \Delta)$ can be represented in form of an integral

$$
\begin{aligned}
\exp (t \Delta)= & (4 \pi t)^{-N / 2} \operatorname{det}_{T M}\left(\frac{\sinh (t \mathcal{B})}{t \mathcal{B}}\right)^{-1 / 2} \exp \left(-t \mathcal{R}^{2}+\frac{1}{6} R_{G} t\right) \\
& \times \int_{\mathbb{R}_{\mathrm{reg}}^{N}} d k|\gamma|^{1 / 2} \operatorname{det}_{\mathcal{G}}\left(\frac{\sinh [C(k) / 2]}{C(k) / 2}\right)^{1 / 2} \\
& \times \exp \left\{-\frac{1}{4 t}\langle k, \gamma t \mathcal{B} \operatorname{coth}(t \mathcal{B}) k\rangle\right\} \exp [\mathcal{L}(k)] .
\end{aligned}
$$

Proof. By using the eq. (2.46) we obtain

$$
\exp (t \Delta)=\exp \left(-t \mathcal{R}^{2}\right) \exp \left(t \mathcal{L}^{2}\right) .
$$

The statement of the theorem follows now from the eqs. (3.47), (3.37), (3.33)(3.36) and (3.14).

\section{Heat Kernel}

\subsection{Heat Kernel Diagonal and Heat Trace}

The heat kernel diagonal on a homogeneous bundle over a symmetric space is parallel. In a parallel local frame it is just a constant matrix. The fiber trace of the heat kernel diagonal is just a constant. That is why, it can be computed at any point in $M$. We fix a point $x^{\prime}$ in $M$ and choose the normal coordinates $y^{a}$ with the origin at $x^{\prime}$ such that the Killing vectors are given by the explicit formulas above (2.15). We compute the heat kernel diagonal at the point $x^{\prime}$. 
The heat kernel diagonal can be obtained by acting by the heat semigroup $\exp (t \Delta)$ on the delta-function, [5, 7]

$$
\begin{aligned}
U^{\mathrm{diag}}(t) & =\left.\exp (t \Delta) \delta\left(x, x^{\prime}\right)\right|_{x=x^{\prime}} \\
& =\left.\exp \left(-t \mathcal{R}^{2}\right) \int_{\mathbb{R}_{\mathrm{reg}}^{N}} d k|G|^{1 / 2} \Phi(t ; k) \exp [\mathcal{L}(k)] \delta\left(x, x^{\prime}\right)\right|_{x=x^{\prime}} .
\end{aligned}
$$

To be able to use this integral representation we need to compute the action of the isometries $\exp [\mathcal{L}(k)]$ on the delta-function.

It is not very difficult to check that the Lie derivatives are nothing but the generators of the pullback, that is,

$$
\mathcal{L}_{\xi} \varphi=k^{A} \mathcal{L}_{A} \varphi=\left.\frac{d}{d t}\left(\psi_{t}^{*} \varphi\right)\right|_{t=0} .
$$

By using this fundamental fact we can prove the following proposition.

Proposition 11 Let $\varphi$ be a section of the twisted spin-tensor bundle $\mathcal{V}, \mathcal{L}_{A}$ be the twisted Lie derivatives, $k^{A}=\left(p^{a}, \omega^{i}\right)$ be the canonical coordinates on the group and $\mathcal{L}(k)=k^{A} \mathcal{L}_{A}$. Let $\xi=k^{A} \xi_{A}$ be the Killing vector and $\psi_{t}$ be the corresponding one-parameter diffeomorphism. Then

$$
\exp [\mathcal{L}(k)] \varphi(x)=\left.\exp \left(-\frac{1}{2} \theta_{a b} G^{a b}\right) \varphi(\hat{x})\right|_{t=1},
$$

where $\hat{x}=\psi_{t}(x)$ and the matrix $\theta$ is defined above by $O=\exp \theta$ where $O$ is defined by (2.61). In particular, for $p=0$ and $x=x^{\prime}$

$$
\left.\exp [\mathcal{L}(k)] \varphi(x)\right|_{p=0, x=x^{\prime}}=\exp [\mathcal{R}(\omega)] \varphi(x) .
$$

Proof. This statement follows from eqs. (2.66) and (2.67).

Proposition 12 Let $\omega^{i}$ be the canonical coordinates on the holonomy group $H$ and $\left(k^{A}\right)=\left(p^{a}, \omega^{i}\right)$ be the natural splitting of the canonical coordinates on the curvature group $G$. Then

$$
\left.\exp [\mathcal{L}(k)] \delta\left(x, x^{\prime}\right)\right|_{x=x^{\prime}}=\operatorname{det}_{T M}\left(\frac{\sinh [D(\omega) / 2]}{D(\omega) / 2}\right)^{-1} \exp [\mathcal{R}(\omega)] \delta(p) .
$$


Proof. Let $\hat{x}\left(t, p, \omega, x, x^{\prime}\right)=\psi_{t}(x)$. By making use of the eq. (4.3) we obtain

$$
\left.\exp [\mathcal{L}(k)] \delta\left(x, x^{\prime}\right)\right|_{x=x^{\prime}}=\left.\exp \left(-\frac{1}{2} \theta_{a b} G^{a b}\right) \delta\left(\hat{x}\left(1, p, \omega, x, x^{\prime}\right), x^{\prime}\right)\right|_{x=x^{\prime}, t=1} .
$$

Now we change the variables from $x^{\mu}$ to the normal coordinates $y^{a}$ to get

$$
\left.\delta\left(\hat{x}\left(1, p, \omega, x, x^{\prime}\right), x^{\prime}\right)\right|_{x=x^{\prime}}=\left.|g|^{-1 / 2} \operatorname{det}\left(\frac{\partial y^{a}}{\partial x^{\mu}}\right) \delta(\hat{y}(1, p, \omega, y))\right|_{y=0} .
$$

This delta-function picks the values of $p$ that make $\hat{y}=0$, which is exactly the functions $\bar{p}=\bar{p}(\omega, y)$ defined above by $\hat{y}(1, \bar{p}, \omega, y)=0$. By switching further to the variables $p$ we obtain

$$
\left.\delta\left(\hat{x}\left(1, p, \omega, x, x^{\prime}\right), x^{\prime}\right)\right|_{x=x^{\prime}}=\left.|g|^{-1 / 2} \operatorname{det}\left(\frac{\partial y^{a}}{\partial x^{\mu}}\right) \operatorname{det}\left(\frac{\partial \hat{y}^{b}}{\partial p^{c}}\right)^{-1} \delta(p-\bar{p}(\omega, y))\right|_{y=0, t=1} .
$$

Now, by recalling that $\left.\bar{p}\right|_{y=0}=0$ and by using the Jacobian $\partial y^{a} / \partial x^{v}$ of the transformation to the normal coordinates in symmetric spaces and (2.53) we evaluate the Jacobians for $p=y=0$ and $t=1$ to get the eq. (4.5).

Remarks. Some remarks are in order here. We implicitly assumed that there are no closed geodesics and that the equation of closed orbits of isometries $\hat{y}^{a}(1, \bar{p}, \omega, 0)=0$ has a unique solution $\bar{p}=\bar{p}(\omega, 0)=0$. On compact symmetric spaces this is not true: there are infinitely many closed geodesics and infinitely many closed orbits of isometries. However, these global solutions, which reflect the global topological structure of the manifold, will not affect our local analysis. In particular, they do not affect the asymptotics of the heat kernel. That is why, we have neglected them here. This is reflected in the fact that the Jacobian in (4.5) can become singular when the coordinates of the holonomy group $\omega^{i}$ vary from $-\infty$ to $\infty$. Note that the exact results for compact symmetric spaces can be obtained by an analytic continuation from the dual noncompact case when such closed geodesics are absent [14]. That is why we proposed above to complexify our holonomy group. If the coordinates $\omega^{i}$ are complex taking values in the subspace $\mathbb{R}_{\text {reg }}^{p}$ defined above, then the equation $\hat{y}^{a}(1, \bar{p}, \omega, 0)=0$ should have a unique solution and the Jacobian is an analytic function. It is worth stressing once again that the canonical coordinates cover the whole group except for a set of measure zero. Also a compact subgroup is covered infinitely many times.

Now by using the above lemmas and the theorem we can compute the heat kernel diagonal. We define the matrix

$$
F(\omega)=\omega^{i} F_{i},
$$


and

$$
R_{H}=-\frac{1}{4} \beta^{i k} F_{i m}^{j} F_{k j}^{m},
$$

which is nothing but the scalar curvature of the isotropy group $H$.

Theorem 8 The heat kernel diagonal of the Laplacian on twisted spin-vector bundles over a symmetric space has the form

$$
\begin{aligned}
U^{\mathrm{diag}}(t)= & (4 \pi t)^{-n / 2} \operatorname{det}_{T M}\left(\frac{\sinh (t \mathcal{B})}{t \mathcal{B}}\right)^{-1 / 2} \exp \left\{\left(\frac{1}{8} R+\frac{1}{6} R_{H}-\mathcal{R}^{2}\right) t\right\} \\
& \times \int_{\mathbb{R}_{\mathrm{reg}}^{n}} \frac{d \omega}{(4 \pi t)^{p / 2}}|\beta|^{1 / 2} \exp \left\{-\frac{1}{4 t}\langle\omega, \beta \omega\rangle\right\} \cosh [\mathcal{R}(\omega)] \\
& \times \operatorname{det}_{\mathcal{H}}\left(\frac{\sinh [F(\omega) / 2]}{F(\omega) / 2}\right)^{1 / 2} \operatorname{det}_{T M}\left(\frac{\sinh [D(\omega) / 2]}{D(\omega) / 2}\right)^{-1 / 2},
\end{aligned}
$$

where $|\beta|=\operatorname{det} \beta_{i j}$ and $\langle\omega, \beta \omega\rangle=\beta_{i j} \omega^{i} \omega^{j}$.

Proof. First, we have $d k=d p d \omega$ and $|\gamma|=|\beta|$. By using the equations (4.1) and (4.5) and integrating over $p$ we obtain the heat kernel diagonal

$$
\begin{aligned}
U^{\mathrm{diag}}(t)= & \int_{\mathbb{R}_{\mathrm{reg}}^{p}} d \omega|G|^{1 / 2}(0, \omega) \Phi(t ; 0, \omega) \operatorname{det}_{T M}\left(\frac{\sinh [D(\omega) / 2]}{D(\omega) / 2}\right)^{-1} \\
& \times \exp \left[\mathcal{R}(\omega)-t \mathcal{R}^{2}\right] .
\end{aligned}
$$

Further, by using the definition of the matrices $C_{A}$ we compute the determinants

$$
\operatorname{det}_{\mathcal{G}}\left(\frac{\sinh [C(\omega) / 2]}{C(\omega) / 2}\right)=\operatorname{det}_{T M}\left(\frac{\sinh [D(\omega) / 2]}{D(\omega) / 2}\right) \operatorname{det}_{\mathcal{H}}\left(\frac{\sinh [F(\omega) / 2]}{F(\omega) / 2}\right) \text {. }
$$

Now, by using (3.31) we compute (3.35) $\Theta(t ; 0, \omega)=\frac{1}{2}\langle\omega, \beta \omega\rangle$, and, finally, by using eq. (3.37), (3.33), (3.13) and (4.10) we get the result (4.11).

By using this theorem we can also compute the heat trace for compact symmetric spaces

$$
\operatorname{Tr}_{L^{2}} \exp (t \Delta)=\int_{M} d \operatorname{vol} \operatorname{tr}_{V} U^{\mathrm{diag}}(t)=\operatorname{vol}(M) \operatorname{tr}_{V} U^{\mathrm{diag}}(t)
$$

where $\operatorname{tr}_{V}$ is the fiber trace. 


\subsection{Heat Kernel Asymptotics}

It is well known that there is the following asymptotic expansion as $t \rightarrow 0$ of the heat kernel diagonal [18]

$$
U^{\mathrm{diag}}(t) \sim(4 \pi t)^{-n / 2} \sum_{k=0}^{\infty} t^{k} a_{k}
$$

The coefficients $a_{k}$ are called the local heat kernel coefficients. On compact symmetric spaces there is a similar asymptotic expansion of the heat trace

$$
\operatorname{Tr}_{L^{2}} \exp (t \Delta) \sim(4 \pi t)^{-n / 2} \sum_{k=0}^{\infty} t^{k} A_{k}
$$

with the global heat invariants $A_{k}$ defined by

$$
A_{k}=\int_{M} d \operatorname{vol} \operatorname{tr}_{V} a_{k}=\operatorname{vol}(M) \operatorname{tr}_{V} a_{k} .
$$

We introduce a Gaussian average over the holonomy algebra by

$$
\langle f(\omega)\rangle=\int_{\mathbb{R}_{\mathrm{reg}}^{p}} \frac{d \omega}{(4 \pi)^{p / 2}}|\beta|^{1 / 2} \exp \left(-\frac{1}{4}\langle\omega, \beta \omega\rangle\right) f(\omega)
$$

Then we can write

$$
\begin{aligned}
& U^{\mathrm{diag}}(t)=(4 \pi t)^{-n / 2} \operatorname{det}_{T M}\left(\frac{\sinh (t \mathcal{B})}{t \mathcal{B}}\right)^{-1 / 2} \exp \left\{\left(\frac{1}{8} R+\frac{1}{6} R_{H}-\mathcal{R}^{2}\right) t\right\} \\
& \times\left\langle\cosh [\sqrt{t} \mathcal{R}(\omega)] \operatorname{det}_{\mathcal{H}}\left(\frac{\sinh [\sqrt{t} F(\omega) / 2]}{\sqrt{t} F(\omega) / 2}\right)^{1 / 2} \operatorname{det}_{T M}\left(\frac{\sinh [\sqrt{t} D(\omega) / 2]}{\sqrt{t} D(\omega) / 2}\right)^{-1 / 2}\right)
\end{aligned}
$$

This equation can be used now to generate all heat kernel coefficients $a_{k}$ for any locally symmetric space simply by expanding it in a power series in $t$. By using the standard Gaussian averages

$$
\begin{aligned}
\left\langle\omega_{1}^{i} \cdots \omega^{i_{2 k+1}}\right\rangle & =0 \\
\left\langle\omega^{i_{1}} \cdots \omega^{i_{2 k}}\right\rangle & =\frac{(2 k) !}{k !} \beta^{\left(i_{1} i_{2}\right.} \cdots \beta^{\left.i_{2 k-1} i_{2 k}\right)},
\end{aligned}
$$

one can obtain now all heat kernel coefficients in terms of traces of various contractions of the matrices $D^{a}{ }_{i b}$ and $F^{j}{ }_{i k}$ with the matrix $\beta^{i k}$. All these quantities are curvature invariants and can be expressed directly in terms of the Riemann tensor. 


\section{Conclusion}

We have continued the study of the heat kernel on homogeneous spaces initiated in [4, 5, 6, 7]. In those papers we have developed a systematic technique for calculation of the heat kernel in two cases: a) a Laplacian on a vector bundle with a parallel curvature over a flat space [4, 6], and b) a scalar Laplacian on manifolds with parallel curvature [5, 7]. What was missing in that study was the case of a non-scalar Laplacian on vector bundles with parallel curvature over curved manifolds with parallel curvature.

In the present paper we considered the Laplacian on a homogeneous bundle and generalized the technique developed in [7] to compute the corresponding heat semigroup and the heat kernel. It is worth pointing out that our formal result applies to general symmetric spaces by making use of the regularization and the analytical continuation procedure described above. Of course, the heat kernel coefficients are just polynomials in the curvature and do not depend on this kind of analytical continuation (for more details, see [7]).

As we mentioned above, due to existence of multiple closed geodesics the obtained form of the heat kernel for compact symmetric spaces requires an additional regularization, which consists simply in an analytical continuation of the result from the complexified noncompact case. In any case, it gives a generating function for all heat invariants and reproduces correctly the whole asymptotic expansion of the heat kernel diagonal. However, since there are no closed geodesics on non-compact symmetric spaces, it seems that the analytical continuation of the obtained result for the heat kernel diagonal should give the exact result for the non-compact case, and, even more generally, for the general case too.

\section{Acknowledgements}

I would like to thank the organizers of the special 2007 Midwest Geometry Conference in the honor of Thomas P. Branson, in particular, Palle Jorgensen, for the invitation and for the financial support. I would like to dedicate this contribution to the memory of Thomas P. Branson (1953-2006) whose sudden death shocked all his friends, collaborators and relatives. Tom was a good friend and a great mathematician who had a major impact on many areas of modern mathematics. 


\section{References}

[1] I. G. Avramidi, Background field calculations in quantum field theory (vacuum polarization), Teor. Mat. Fiz., 79 (1989), 219-231.

[2] I. G. Avramidi, The covariant technique for calculation of the heat kernel asymptotic expansion, Phys. Lett. B, 238 (1990), 92-97.

[3] I. G. Avramidi, A covariant technique for the calculation of the one-loop effective action, Nucl. Phys. B 355 (1991) 712-754; Erratum: Nucl. Phys. B 509 (1998) 557-558.

[4] I. G. Avramidi, A new algebraic approach for calculating the heat kernel in gauge theories, Phys. Lett. B, 305 (1993), 27-34.

[5] I. G. Avramidi, The heat kernel on symmetric spaces via integrating over the group of isometries, Phys. Lett. B 336 (1994) 171-177.

[6] I. G. Avramidi, Covariant algebraic method for calculation of the low-energy heat kernel, J. Math. Phys. 36 (1995) 5055-5070; Erratum: J. Math. Phys. 39 (1998) 1720.

[7] I. G. Avramidi, A new algebraic approach for calculating the heat kernel in quantum gravity, J. Math. Phys. 37 (1996) 374-394.

[8] I. G. Avramidi, Covariant techniques for computation of the heat kernel, Rev. Math. Phys., 11 (1999), 947-980.

[9] I. G. Avramidi, Heat Kernel and Quantum Gravity, Lecture Notes in Physics, Series Monographs, LNP:m64, Springer-Verlag, Berlin, 2000.

[10] I. G. Avramidi, Heat kernel approach in quantum field theory, Nucl. Phys. Proc. Suppl., 104 (2002), 3-32.

[11] I. G. Avramidi, Heat kernel asymptotics on symmetric spaces, Int. J. Geom. Topol., (2007) (to be published); arXiv:math.DG/0605762

[12] I. G. Avramidi, Heat kernel on homogeneous bundles over symmetric spaces, arXiv:math.AP/0701489, 55 pp., submitted to Advances in Math. (2007),

[13] A. O. Barut and R. Raszka, Theory of Group Representations and Applications, PWN, Warszawa, 1977.

[14] R. Camporesi, Harmonic analysis and propagators on homogeneous spaces, Phys. Rep. 196, (1990), 1-134.

[15] J. S. Dowker, When is the "sum over classical paths" exact?, J. Phys. A, 3 (1970), 451-461.

[16] J. S. Dowker, Quantum mechanics on group space and Huygen's principle, Ann. Phys. (USA), 62 (1971), 361-382.

[17] P. B. Gilkey, The spectral geometry of Riemannian manifold, J. Diff. Geom., 10 (1975), 601-618.

[18] P. B. Gilkey, Invariance Theory, the Heat Equation and the Atiyah-Singer Index Theorem, CRC Press, Boca Raton, 1995. 
[19] S. Helgason, Groups and Geometric Analysis: Integral Geometry, Invariant Differential Operators, and Spherical Functions, Mathematical Surveys and Monographs, vol. 83, AMS, Providence, 2002, p. 270

[20] K. Kirsten, Spectral Functions in Mathematics and Physics, CRC Press, Boca Raton, 2001.

[21] H. Ruse, A. G. Walker and T. J. Willmore, Harmonic Spaces, Edizioni Cremonese, Roma, 1961.

[22] M. Takeuchi, Lie Groups II, in: Translations of Mathematical Monographs, vol. 85, AMS, Providence, 1991, p.167.

[23] A. E. M. Van de Ven, Index free heat kernel coefficients, Class. Quant. Grav. 15 (1998), 2311-2344.

[24] D. V. Vassilevich, Heat kernel expansion: user's manual, Phys. Rep., 388 (2003), 279-360.

[25] J. A. Wolf, Spaces of Constant Curvature, University of California, Berkeley, 1972.

[26] S. Yajima, Y. Higasida, K. Kawano, S.-I. Kubota, Y. Kamo and S. Tokuo, Higher coefficients in asymptotic expansion of the heat kernel, Phys. Rep. Kumamoto Univ., 12 (2004), No 1, 39-62. 